\title{
AN ASSESSMENT OF THE GENETIC DIVERSITY IN SELECTED WHEAT LINES USING MOLECULAR MARKERS AND PCA- BASED CLUSTER ANALYSIS
}

\author{
ALI, Y. $.^{*}-$ KHAN, M. A. ${ }^{1}-$ HUSSAIN, M. ${ }^{2}-$ AtIQ, M. ${ }^{1}-$ AHMAD, J. N. ${ }^{3}$ \\ ${ }^{I}$ Department of Plant Pathology, University of Agriculture Faisalabad, Faisalabad, Pakistan \\ ${ }^{2}$ Plant Pathology Research Institute, Ayub Agricultural Research Institute, Faisalabad, Pakistan \\ ${ }^{3}$ Department of Entomology, University of Agriculture Faisalabad, Faisalabad, Pakistan \\ *Corresponding author \\ e-mail: yasirklasra.uca@gmail.com \\ (Received $7^{\text {th }}$ Jul 2018; accepted 20 $0^{\text {th }}$ Sep 2018)
}

\begin{abstract}
A comprehensive germplasm evaluation study of wheat elite lines was conducted at Wheat Research Institute Faisalabad-Pakistan to identify new sources of leaf, stripe and stem rust resistance and high yield potential during crop seasons 2015-2017. The parent lines were selected on the basis of phenotypic characteristics and slow rusting history for race non-specific resistance genes by the selection of desirable parents used in filial generation (F1-F5). In primary evaluation, 112 lines were selected on the basis of rust reaction and high phenotypic uniformity for further testing against rust resistance and high yield potential. Among these, 32 lines exhibited Lr34/Yr18, 22 lines showed $L r 46 / Y r 29$, and 30 lines indicated the combination of $S r 2 / Y r 30$. Principal component analysis (PCA) based cluster analysis exhibited that, cluster I and III had clear separation compared to cluster II, IV and V. It was concluded that seven elite lines i.e. V-70003, V-70034, V-70054, V-70070, V-70085, V-70103 and V-70104 exhibited both the linkages of three slow rusting genes ( $\operatorname{Lr} 34 / \mathrm{Yr} 18, \mathrm{Lr} 46 / \mathrm{Yr} 29$ and $\mathrm{Sr} 2 / \mathrm{Yr} 30$ ) and high yield characteristics and are expected to contribute toward food security at national and global levels.
\end{abstract}

Keywords: breeding, grain yield, rust resistance, SSR markers, Triticum aestivum

\section{Introduction}

Wheat (Triticum aestivum L.) along with maize and rice is a strategic crop for worldwide food security. The estimated global wheat production for the year 2015-2016 is 734.2 MT which is slightly higher than the demand of 716.2 MT (FAO, 2016). The demand for wheat continues to rise at an annual rate of $1.6 \%$ and some estimates indicate that $60 \%$ more wheat will be needed by 2050 (FAO, 2016).

Wheat is mainly hit by three types of rusts stripe/yellow, leaf/brown and stem/black that reduce its produce (Roelfs et al., 1992). Evolution of two high temperature tolerant yellow rust races caused severe epidemics in main wheat growing regions of the world since 2000 (Hovmoller et al., 2008). Recent identification of various virulent races of Ug99 i.e. TTKSK, TTKSF, TTKSF+, TTKSP, PTKST and three virulent brown rust races CCPS, MCDS and FBPT are significant threat to wheat production worldwide necessitating integrated and collaborative management strategies of the diseases (Terefe et al., 2014; Pretorius et al., 2015; Patpour et al., 2016).

In Pakistan yellow and leaf rust have been a constant risk to its sustainable production. The reason behind rapid collapse of the assortments is associated to the evolution of new virulent races in assortment due to race specific genes of presentation. The recent and last trend of genomic fight in wheat assortment is "resistance based on preservative effects of accumulation of race non-specific genes" (Singh et al., 1998). 
The race non-specific yellow and leaf rust resistance appearing in several assortments is based on durable genes that have additive effects (Singh et al., 2005). The economic, most effective, environmentally friendly, and easy to use method to reduce losses caused by the rusts is cultivation of resistant assortments (Cheng and Chen, 2014; Kalappanavar et al., 2008). In current era of scientific research main focus is to achieve race non-specific slow rusting resistance by combining several minor or adult plant genes (Singh et al., 2000).

Continuous breeding results in narrow genetic variation in gene pool of wheat advance lines and also lead to problems regarding adaptation as well as biotic and abiotic stresses (Zhang et al., 2005). Highest genetic variation among parentage is necessary to achieve transgressive segregation (Joshi et al., 2004). Selection of genetically different parentage through breeding results in maximum variation in progenies. Therefore, there is an urgent need to exploit the existing elite lines to evolve high yielding lines that have extensive adoptability under changing meteorological conditions (Baranwal et al., 2012). The use of molecular markers for the assessment of genetic variation is receiving much attention. Many wheat researchers have studied the genetic diversity in bread wheat using different molecular markers such as RFLPs (Kim and Ward, 2000), ISSRs (Nagaoka and Ogihara, 1997), STS (Chen et al., 1994), AFLPs (Burkhamer et al., 1998) and RAPDs (Joshi and Nguyen, 1993). Though, the most of these molecular marker systems (Devos and Gale, 1992) exhibit a low level of genetic diversity in the selected wheat lines, especially among cultivated lines/cultivars.

The simple sequence repeats (SSRs), also termed as microsatellites, have been proposed as the most-suitable markers for the evaluation of diversity and genetic variation among wheat cultivars/lines, as they are chromosome-specific, multiallelic and consistently distributed along chromosomes (Roder et al., 1998). The SSRs markers have been applied widely for genetic stability of gene bank accessions (Borner et al., 2000), marker-assisted selection in wheat (Huang et al., 2000), identifying QTLs (Kandel et al., 2017), and tagging resistance genes (Mutari et al., 2018). Such molecular markers have also demonstrated a high level of genetic diversity among diploid species (Hammer et al., 2000). Such markers also revealed a high level of polymorphism among diploid species (Hammer et al., 2000), in the accessions of tetraploid wild wheat Triticum dicoccoides (Fahima et al., 2002), and as well as in hexaploid wheat varieties (Stachel et al., 2000; Prasad et al., 2000). Cluster and principal component analyses are main genomic diversity tools having comparative differences with each other. PCA based cluster analysis is robust technique to assess family linkage (Mellingers, 1972). Hence, the main goal of present study were to evaluate (1) wheat advanced lines having race non-specific rust resistance through DNA molecular markers (2) high yielding lines through cluster and Principal component analyses.

\section{Materials and methods}

For genetic evaluation plant material comprised 855 wheat elite lines (F6 generation) of 45 diverse crosses based on 8-10 year wheat rust history and high yield characteristics (Table 1) were selected from gene pool of Wheat Research Institute Faisalabad. The trial was sown during $2^{\text {nd }}$ week of November, 2015-2016 at Wheat Research Institute (WRI) Faisalabad through hand drill following augmented design with single replication split with 9 blocks having five plots per block containing 19 genotypes with one check (Morocco). Each plot comprises of 20 rows $2.5 \mathrm{~m}$ long and 
$25 \mathrm{~cm}$ apart. Morocco was inoculated using spraying, dusting and hypodermal needle injection methods twice during month of January and February to develop high rust inoculum pressure (Roelfs, 1988). Disease severity percentage and field response were observed following modified Cobb's scale (Table 2) for five consecutive observations after every 7 days interval when morocco became 50-60\% susceptible.

Table 1. Detail of parents used in crossing

\begin{tabular}{|c|c|c|c|c|c|}
\hline $\mathbf{S} / \mathbf{N}$ & Name of line/cultivar & $\begin{array}{c}\text { Leaf rust resistance } \\
\text { status }\end{array}$ & $\begin{array}{c}\text { Stripe rust } \\
\text { resistance status }\end{array}$ & $\begin{array}{l}\text { Acceptable } \\
\text { yield kg ha }\end{array}$ & $\begin{array}{l}\text { Maximum } \\
\text { yield kg ha- }\end{array}$ \\
\hline 1 & INQ.91 & Moderately resistant & Moderately resistant & 4800 & 6700 \\
\hline 2 & WBLLI & Resistant & Resistant & 4250 & 6850 \\
\hline 3 & AS-2002 & Moderately resistant & Susceptible & 4550 & 6655 \\
\hline 4 & FSD.08 & Partially resistant & Moderately resistant & 4453 & 6650 \\
\hline 5 & $\begin{array}{c}\text { AUQAB } \\
2000 * 2 / \text { LAKTA- } 1\end{array}$ & Resistant & Resistant & 4775 & 6900 \\
\hline 6 & V-87094 & Partially resistant & Partially resistant & 4850 & 6900 \\
\hline 7 & V-09014 & Slow rusting & Susceptible & 4700 & 6850 \\
\hline 9 & SH.88/PAK.81 & Partially Resistant & Resistant & 4611 & 6800 \\
\hline 10 & SHAFAQ-06 & Partially resistant & Partially resistant & 4100 & 6400 \\
\hline 11 & MILAN/KAUZ & Resistant & Susceptible & 4011 & 6100 \\
\hline 12 & BABAX & Partially resistant & Partially resistant & 4100 & 6700 \\
\hline 13 & ALTAR & Moderately resistant & Resistant & 4310 & 6850 \\
\hline 14 & MAYA 74'S'/MON'S' & Susceptible & Partially resistant & 4300 & 6500 \\
\hline 15 & MAYA/PVN & Resistant & Resistant & 4011 & 6430 \\
\hline 17 & PB96/V87094//MH97 & Moderately resistant & Resistant & 4204 & 6100 \\
\hline 18 & TRAP\#1 & Resistant & Resistant & 4500 & 6600 \\
\hline 19 & SH88/2*ATTILA & Moderately resistant & $\begin{array}{l}\text { Moderately } \\
\text { susceptible }\end{array}$ & 4800 & 6700 \\
\hline 20 & CNDO/R143 & Resistant & Resistant & 4250 & 6850 \\
\hline 21 & SERI.1B & Moderately resistant & Susceptible & 4550 & 6655 \\
\hline 22 & PBW343*2/KUKUNA & Partially resistant & Moderately resistant & 4453 & 6650 \\
\hline 23 & C80.1/3*BATAVIA & Susceptible & Resistant & 4475 & 6800 \\
\hline 24 & WH576 & Partially resistant & Partially resistant & 4750 & 6700 \\
\hline 25 & PASTOR & Resistant & Resistant & 3800 & 6450 \\
\hline 26 & MEXI_2 & Partially resistant & Partially resistant & 4200 & 6600 \\
\hline 27 & KRONSTADF2004 & Moderately resistant & Resistant & 4310 & 6760 \\
\hline 28 & ROLF07*2/KIRITATI & Susceptible & Partially resistant & 4200 & 6650 \\
\hline 29 & KIRITATI & Resistant & Resistant & 4050 & 6050 \\
\hline 30 & WAXWING & Slow rusting & Susceptible & 4000 & 6150 \\
\hline
\end{tabular}

The genotypes recorded to be resistant through primary evaluation (112) along with five checks were subjected to further screening for rusts resistance and high yield potential at WRI Faisalabad during second week of November, 2016-2017. The genotypes were planted by Norvigion in experimental area of Wheat Research Institute in Augmented design. Each test entry was planted in a plot (six rows of five meter 
length). In order to facilitate development of rust epidemics two rows of Morroco were planted around each side of experimental material. Artificial inoculation of experimental material was done in the morning from first week of January to end of February using spraying, dusting and hypodermal needle injection method (Rao et al., 1989), twice a week until a heavy inoculum develops (Roelfs et al., 1992). The applied inoculum consisted of yellow (80E85) and mixture of leaf rust (PHTTL, PGRTB, KSR/JS, TKTPR and TKTRN) races collected from Murree, Kaghan and Faisalabad. High humidity was maintained by frequent irrigations.

Table 2. Disease rating scale used for rust resistance/susceptibility of wheat elite lines

\begin{tabular}{|c|c|c|}
\hline Reaction & Code & Symptoms \\
\hline Immune & 0 & No visible infection \\
\hline Resistant & $\mathrm{R}$ & Visible necrotic or chlorosis with or without uredia \\
\hline Moderately resistant & MR & Small uredia surrounded by necrotic areas \\
\hline Mixed (intermediate) & M & $\begin{array}{l}\text { Small uredia present surrounded by necrotic areas as well as medium } \\
\text { uredia with no necrosis but possible some distinct chlorosis }\end{array}$ \\
\hline Moderately susceptible & MS & Medium uredia with no necrosis but possible some distinct chlorosis \\
\hline $\begin{array}{l}\text { Moderately susceptible- } \\
\text { susceptible }\end{array}$ & MSS & $\begin{array}{l}\text { Medium uredia with no necrosis but possible some distinct chlorosis as } \\
\text { well as large uredia with little or chlorosis present }\end{array}$ \\
\hline Susceptible & S & Large uredia are present with little or no chlorosis \\
\hline
\end{tabular}

Cobb's scale (Peterson et al., 1948)

\section{Molecular evaluation and yield testing of 112 selected wheat advance lines through molecular marker and PCA based cluster analysis}

The putatively selected 112 wheat advance lines through primary evaluation were further assayed to molecular characterization to identify race non-specific resistance genes $L r 34 / Y r 18$, Lr46/Yr29 and Sr2/Yr30 using a set of 3 DNA molecular markers viz. X-barc-352, XWMC-44, and Xgwm-533 respectively (William et al., 2003; Suenaga et al., 2003; Hussain et al., 2015). This present study work was carried out at Integrated Genomics Cellular Developmental and Biotechnology Laboratory, Post Graduate Agricultural Research Station (PARS) Campus, University of Agriculture Faisalabad.

\section{DNA extraction and quantification}

The fresh leaf samples from 30 day-old seedling were collected from the Wheat Research Institute Faisalabad. After tagging, samples were washed with purified water and frozen immediately in liquid nitrogen $\left(\mathrm{LN}_{2}\right)$ chamber available in PARS campus University of Agriculture-Faisalabad and stored at $-80{ }^{\circ} \mathrm{C}$ in deep freeze for DNA extraction by using Cetyl Trimethyl Ammonium Bromide (CTAB) method (Bansal et al., 2014). Leaves were crushed in CTAB buffer to release DNA from the cell. Samples were incubated in water bath at $65{ }^{\circ} \mathrm{C}$ for $25-30 \mathrm{~min}$. Tubes were centrifuge at $4000 \mathrm{rpm}$ for $5 \mathrm{~min}$ and the upper aqueous phase was transferred to new tubes. Chloroform: isoamyl alcohol (24:1 v/v) $(300-500 \mu \mathrm{l})$ was added and vortex 4-5 times to mix the contents properly. For further purification other reagents such as RNase and $\mathrm{NaCl}$ were also added and centrifuged for $5 \mathrm{~min}$ at $14000 \mathrm{rpm}$ and supernatant was transferred to fresh tubes. DNA was precipitated by adding $(500 \mu \mathrm{l})$ of chilled isopropanol in the tubes and let it at $-20{ }^{\circ} \mathrm{C}$ for $25-30 \mathrm{~min}$. The tubes were then 
centrifuged at $14000 \mathrm{rpm}$ for $15 \mathrm{~min}$ to precipitate the DNA. The DNA pellet was washed 2-3 times with $500 \mu \mathrm{l}$ of $70 \%$ ethanol and air dried before re-suspension in 20$30 \mu \mathrm{ldd} \mathrm{H}_{2} \mathrm{O}$. The DNA concentration was measured by spectrophotometer. An aliquot of sample was diluted in water $\left(1 / 80^{\text {th }}\right.$ or $\left.1 / 100^{\text {th }}\right)$ and its absorbance was measured at $260 \mathrm{~nm}$ using a UV spectrophotometer.

\section{PCR-marker assay}

PCR amplifications were performed in a total volume of $25 \mu 1$ containing 50$100 \mathrm{ng} / \mu \mathrm{l}$ of genomic DNA, $2.5 \mu \mathrm{l}$ of 10X PCR buffer with $2.5 \mathrm{mM}(2 \mu \mathrm{l})$ of Mgcl2, 0.5 of $10 \mathrm{mM}$ dNTPS, $0.5 \mu \mathrm{l}$ each forward and reverse primer, $1 \mathrm{U}$ of Taq DNA polymerase and $17 \mu \mathrm{l}$ of ddH20 (Ahmad et al., 2017). Reagents were purchased from Invitrogen (USA). PCR was performed using the Eppendorf Mastercycler, Germany. The amplification parameters used for all primer sets i.e. X-barc-352, Xwmc-44 and Xgwm533 restricted to specific durable resistance genes are presented in Table 3.

Table 3. Amplification parameters used for all primer sets linked to their specific durable resistance genes

\begin{tabular}{|c|c|c|}
\hline Resistance genes & Primers & Cycle condition \\
\hline Lr34/Yrl8 & X-barc-352 & $\begin{array}{c}94^{\circ} \mathrm{C} 5 \mathrm{~min}, 38 \text { cycles }\left(94^{\circ} \mathrm{C} 30 \mathrm{~s}, 60^{\circ} \mathrm{C} 30 \mathrm{~s}-1 \mathrm{~min}, 72^{\circ} \mathrm{C} 30 \mathrm{~s}\right), \\
72{ }^{\circ} \mathrm{C} 5 \mathrm{~min}\end{array}$ \\
\hline$L r 46 / Y r 29$ & Xwmc-44 & $\begin{array}{c}94{ }^{\circ} \mathrm{C} 5 \min , 45 \text { cycles }\left(94{ }^{\circ} \mathrm{C} 1 \mathrm{~min}, 55^{\circ} \mathrm{C} 1 \mathrm{~min}, 72^{\circ} \mathrm{C} 2 \mathrm{~min}\right), 72 \\
{ }^{\circ} \mathrm{C} 10 \mathrm{~min}\end{array}$ \\
\hline Sr2/Yr30 & Xwm-533 & $\begin{array}{c}94{ }^{\circ} \mathrm{C} 5 \min , 45 \text { cycles }\left(94{ }^{\circ} \mathrm{C} 1 \min , 60^{\circ} \mathrm{C} 1 \mathrm{~min}, 72{ }^{\circ} \mathrm{C} 2 \mathrm{~min}\right), 72 \\
{ }^{\circ} \mathrm{C} 10 \mathrm{~min}\end{array}$ \\
\hline
\end{tabular}

\section{Electrophoresis}

After PCR amplification, electrophoresis was carried out on the Syngene gel documentation system USA for SSR markers (Hussain et al., 2015). An amount of $1.5 \mathrm{~g}$ high resolution agarose gel was weighted in the electric balance and dissolved in $100 \mathrm{~mL} 1 \mathrm{X}$ TAE buffer (acetic acid $\mathrm{pH}=7.8$; Sodium acetate $2 \mathrm{mM}$; EDTA $10 \mathrm{mM}$; Tris HCL $40 \mathrm{mM}$ ) in a conical flask. It was heated for about 2-3 min by keeping it in oven and then left to cool under running tap water and mixed gently after adding $2 \mu 1$ ethidium bromide (fluorescent dye) in this solution. The prepared solution was poured slowly into the gel tank. The combs of required size and teeth were inserted in it and leave it for 10-15 min to allow polymerization of gel. After polymerization, the 1XTAE buffer was poured into the gel tank to submerge the gel to 3-6 mm depth. The first well was loaded with $1 \mathrm{~Kb}$ ladder molecular weight marker (Promega) as a size standard. Appropriate amounts of about $8 \mu \mathrm{L}$ of each PCR samples were loaded into the other wells. The gel tank was closed and the gel was run for 30 min by providing 50 to $100 \mathrm{~V}$ current to intercalate ethidium bromide in gel. After electrophoresis, the amplified products were visualized under ultraviolet transilluminator and gel pictures were obtained using Gene Snap version 7.6.03 of Syngene gel documentation system USA.

\section{Yield testing of selected advanced lines on the basis of their genetic traits}

For yield testing, data of other genetic traits such as plant height $(\mathrm{cm})$, spike length $(\mathrm{cm})$, the number of spikelet per spike, yield $\left(\mathrm{kg} \mathrm{ha}^{-1}\right)$, thousand grain weight (gram) 
and protein percentage of all the 112 selected advanced lines along with five checks were recorded. The combined data of grain yield and its components were then subjected to analysis to estimate mean, standard error, range, simple correlation and variance. All variables traits were analyzed by PCA based cluster analyses using software program Statistca v. 10 and SPSS v.12. Cluster analysis identifies parameters which are further classified into a number of clusters following Ward's methods (Ali et al., 2008). The lines in each cluster were also analyzed for simple statistics. To show variation pattern among lines Euclidean distance were calculated and their relationship was shown in the scattered diagram.

\section{Results}

The current study was planned to achieve durable-type resistance by accumulating designated slow rusting race non-specific genes with high yield characteristics of wheat advance lines. The plant material was selected from 855 heads rows of 45 crosses planted at Wheat Research Institute Faisalabad, during crop 2015-2016, only 112 lines were selected on the basis of grain color, shape, high phenotypic uniformity and rust reactions (Table 4).

Table 4. Selection of single head crosses from F6 generation of 45 crosses during 2015-2016

\begin{tabular}{|c|c|c|c|}
\hline Sr.\# & Name of crosses & $\begin{array}{l}\text { Tested } \\
\text { entries }\end{array}$ & $\begin{array}{c}\text { Selected } \\
\text { entries }\end{array}$ \\
\hline 1 & $\begin{array}{l}\text { CHENAB2000/INQ.91/5/WBLL1 } * 2 / 4 / \mathrm{SNI} / \mathrm{TRAP} \# 1 / 3 / \mathrm{KAUZ} * 2 / \mathrm{TRAP} / / \\
\text { KAUZ }\end{array}$ & 19 & 3 \\
\hline 2 & AS-2002/5/FRET2*2/4/SNI/TRAP\#1/3/KAUZ*2/TRAP//KAL & 19 & 1 \\
\hline 3 & FSD.08/6/BABAX/3/FASAN/Y//KAUZ/4/BABAX/5/LU 26/HD2179 & 19 & 4 \\
\hline 4 & KAUZ//ALTAR84/AOS/3/MILAN/KAUZ/4/HUITES/5/KAUZ//ALTAR & 19 & 8 \\
\hline 5 & SH.88/PAK.81//MH.97//OTUS/TOBA97 & 19 & 3 \\
\hline 6 & SH.88/PAK.81//MH.97//CUMHURIYET/NE & 19 & 3 \\
\hline 7 & OASIS/5*ANGRA//INQ.91///MILAN/S87230//BABAX & 19 & 4 \\
\hline 8 & TRM//MAYA 74'S'/MON'S'/3/INQ.91/4/PBW343 & 19 & 7 \\
\hline 9 & 87094/ERA//PAK-81/2*V-87094/3/SHAFAQ-06/4/MAYA/PVN & 19 & 5 \\
\hline 10 & $\begin{array}{l}\text { PFAU/MILAN/5/CHEN/A.SQ(TAUS)//BCN/3/VEE\#7/BOW/4/PASTOR } \\
\text { /6/QINGHAIBRI/WBLLI//BRBT2 }\end{array}$ & 19 & 3 \\
\hline 11 & INQALAB 91*2/KUKUNA//KIRITATI///V-09014 & 19 & 3 \\
\hline 12 & AUQAB $2000 * 2 / L A K T A-1$ & 19 & 4 \\
\hline 13 & $\begin{array}{l}\text { FSD.08/6/BABAX/3/FASAN/Y//KAUZ/4/BABAX/5/LU26/HD2179/7/P } \\
\text { B.96/87094//MH.97 }\end{array}$ & 19 & 6 \\
\hline 14 & $\begin{array}{l}\text { TAM200/Tui/6/PVN/CRC422/ANA/5/BOW//CROW/BUC/PVN/3/YR/Y } \\
\text { R/4/TRAP\#1/7/*21NQ-91 }\end{array}$ & 19 & 2 \\
\hline 15 & INQ/AUQAB/3/SH.88/90A204//MH.97 & 19 & 1 \\
\hline 16 & SH88/WEAVER/3/DWL5023/SNB//SNB & 19 & 1 \\
\hline 17 & $\begin{array}{l}\text { SH88/WEAVER/6/LU26/HD2179/5/BABAX/3/MANGO/VEE\#10//PRL } \\
\text { /4/BABAX }\end{array}$ & 19 & 0 \\
\hline 18 & $\begin{array}{l}\text { KAUZ//ALTAR84/AOS/3/PASTOR/4/TILHI/7/CNO79//PF70354/MUS/ } \\
\text { 3/PASTOR/4/BAV92/5/FRET2/KUKUNA//FRET2/6/MILAN/KAUZ//P } \\
\text { RINIA/3/BAV92 }\end{array}$ & 19 & 0 \\
\hline 19 & $\begin{array}{c}\text { SH88/2*ATTILA/6/ACHTAR*3//KANZ/KS8585/4/MILAN/KAUZ//PRI } \\
\text { NIA/3/BAV92/5/MILAN/KAUZ//PRINIA/3/BAV92 }\end{array}$ & 19 & 2 \\
\hline
\end{tabular}




\begin{tabular}{|c|c|c|c|}
\hline 20 & $\begin{array}{c}\text { CNDO/R143//ENTE/MEXI_2/3/AEGILOPSSQUARROSA(TAUS)/4/W } \\
\text { EAVER/5/PICUS/6/TROST/7/TACUPETO } \\
\text { F2001/8/OASIS/SKAUZ//4*BCN/3/2*PASTOR }\end{array}$ & 19 & 3 \\
\hline 21 & $\begin{array}{c}\text { CNDO/R143//ENTE/MEXI_2/3/AEGILOPSSQUARROSA(TAUS)/4/W } \\
\text { EAVER/5/PICUS/6/TROST/7/TACUPETOF2001/8/CROW'S'/NAC//BO } \\
\text { W'S' } \\
\end{array}$ & 19 & 9 \\
\hline 22 & $\begin{array}{c}\text { PFAU/SERI.1B//AMAD/3/INQALAB91 *2/KUKUNA/4/WBLL1*2/KUR } \\
\text { UKU/5/PVN/YACO/3/KAUZ*2/TRAP// KAUZ }\end{array}$ & 19 & 3 \\
\hline 23 & $\begin{array}{c}\text { HUW234+LR34/PRINIA//PBW343*2/KUKUNA/3/ROLF07/4/SNI/TRA } \\
\text { P\#1/3/KAUZ*2/TRAP//KAUZ }\end{array}$ & 19 & 0 \\
\hline 24 & $\begin{array}{c}\mathrm{PRL} / 2 * \mathrm{PASTOR} / / \mathrm{PBW} 343 * 2 / \mathrm{KUKUNA} / 4 / \mathrm{CAR} 422 / \mathrm{ANA} / / \mathrm{TRAP} \# 1 / 3 / \mathrm{K} \\
\mathrm{AUZ} * 2 / \mathrm{TRAP} / / \mathrm{KAUZ}\end{array}$ & 19 & 0 \\
\hline 25 & $\begin{array}{l}\text { C80.1/3*BATAVIA//2*WBLL1/3/PBW343*2/KUKUNA/4/KAUZ / } \\
\text { SITE }\end{array}$ & 19 & 3 \\
\hline 26 & $\begin{array}{l}\text { INQALAB } 91 * 2 / \mathrm{KONK} / / \mathrm{INQALAB} 91 * 2 / \mathrm{KUKUNA} / 3 / \mathrm{INQ}- \\
91 * 2 / \mathrm{TUKURU}\end{array}$ & 19 & 1 \\
\hline 27 & WHEAR/KRONSTAD F2004/3/CROW'S'/NAC//BOW'S' & 19 & 1 \\
\hline 28 & WHEAR/KRONSTADF2004/3/PB96/V87094//MH97 & 19 & 1 \\
\hline 29 & FRT/SA42/3/PB96/87094//MH-97 & 19 & 1 \\
\hline 30 & WHEAR/KRONSTAD F2004//KAUZ / SITE & 19 & 7 \\
\hline 31 & $\begin{array}{l}\text { PFAU/MILAN//PBW343*2/TUKURU/3/T.DICOCCON P194625/A.SQ } \\
(372) / / \text { TUI.... }\end{array}$ & 19 & 1 \\
\hline 32 & PFAU/MILAN//PBW343*2/TUKURU/3/NR381 & 19 & 1 \\
\hline 33 & $\begin{array}{c}\text { CROC_1/AE.SQUARROSA(205)//KAUZ/3/ATTILA/4/BOW/PRL//BUC } \\
\text { /3/WH576/5/AMSEL/ATTILA//INQ.91/PEW'S' }\end{array}$ & 19 & 3 \\
\hline 34 & $\begin{array}{c}\text { CROC_1/AE.SQUARROSA } \\
(205) / / \text { KAUZ/3/PASTOR/4/THELIN/5/INQ/AUQAB }\end{array}$ & 19 & 5 \\
\hline 35 & MINO/898.97/4/INIA66/7C//MAYA/3/PCI/TRM & 19 & 1 \\
\hline 36 & CHONTE//PBW343*2/KUKUNA/3/CHENAB2000/INQ.91 & 19 & 0 \\
\hline 37 & $\begin{array}{c}\text { CHONTE//PBW343*2/KUKUNA/INQ.91*2/TUKURU/3/T.DICOCCOM } \\
\text { /P194624/AE.SQ (409)//BCN/4/2*INQ.91/2*/... }\end{array}$ & 19 & 1 \\
\hline 38 & PB96/87094/MH-97/3/AMSEL/ATTILA//INQ.91/PEW'S' & 19 & 0 \\
\hline 39 & PB96/87094//MH-97/3/MILAN/S87230//BABAX & 19 & 1 \\
\hline 40 & LU26/HD2179//TTR'S'/JUN'S'/3/HP1744//4/MILAN/S87230//BABAX & 19 & 0 \\
\hline 41 & $\begin{array}{c}\text { CNDO/R143//ENTE/MEXI_2/3/AEGILOPSSQUARROSA(TAUS)/4/W } \\
\text { EAVER/5/IRENA/6/LERKE/7/TAN/PEW//SARA/3/CBRD }\end{array}$ & 19 & 5 \\
\hline 42 & $\begin{array}{c}\text { PBW343*2/KUKUNA//KRONSTADF2004/3/PBW343*2/KUKUNA/4/C } \\
\text { HENAB2000/INQ.91 }\end{array}$ & 19 & 1 \\
\hline 43 & $\begin{array}{c}\text { PBW343*2/KUKUNA//KRONSTADF2004/3/PBW343*2/KUKUNA/4/C } \\
\text { HENAB2000/INQ.91 }\end{array}$ & 19 & 1 \\
\hline 44 & ATTILA*2//CHIL/BUC*2/3/KUKUNA/4/WAXWING*2/TUKURU & 19 & 1 \\
\hline \multirow[t]{2}{*}{45} & ROLF07*2/KIRITATI/3/SW8688//PBW343*2/KUKUNA & 19 & 2 \\
\hline & Total & 855 & 112 \\
\hline
\end{tabular}

All 112 selected wheat elite lines were further characterized on the basis of amplification of SSR molecular markers X-Barc352, Xwmc-44 and Xgwm-533 (Table 5). Among these lines, 32 lines exhibited Lr34/Yr18, 22 lines showed Lr46/Yr29, and 30 lines indicated the combination of Sr2/Yr3. Molecular marker X-barc-352 indicated association to $\mathrm{Lr} 34 / \mathrm{Yr} 18$ which was present on chromosomal loci 1BL. Only 
24 advanced lines were amplified by polymerase chain reaction (PCR) in which 19 genotypes were resistant and five advanced lines i.e. V-70001, V-70005, V-70006, V70008, V-70009 and V-70010 were found susceptible (Fig. 1).

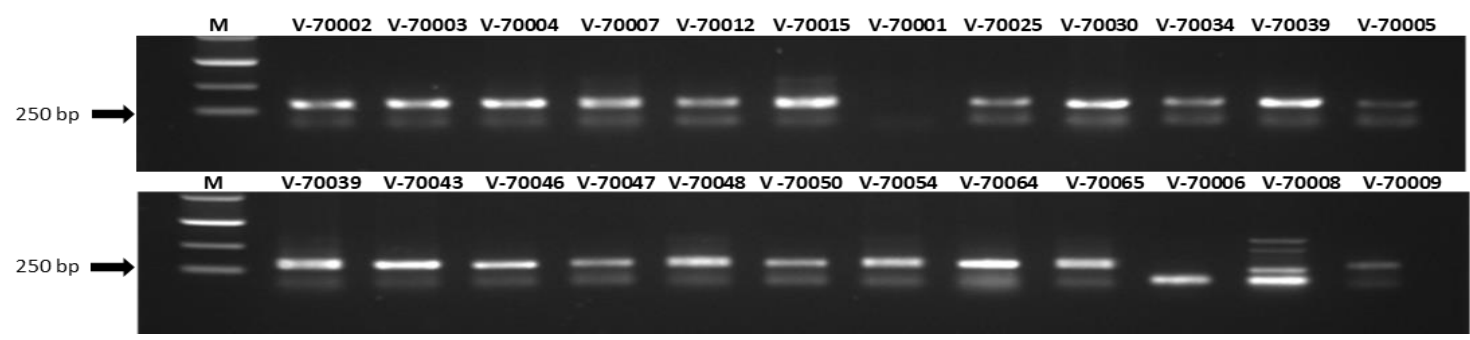

Figure 1. PCR amplification profile of 24 advanced lines for SSR marker X-barc 352 linked to Lr34/Yr18; $M=1 \mathrm{~Kb}$ DNA Ladder Marker

Table 5. Detail of selected elite lines showing combination of three designated slow ruster, race non-specific resistance genes

\begin{tabular}{|c|c|c|c|c|}
\hline \multicolumn{2}{|r|}{ Plant material } & \multicolumn{3}{|c|}{ Genotypic markers } \\
\hline V. Code & Name of genotypes & $\begin{array}{c}\text { Lr34/Yrl8 } \\
\text { (X-barc-352) }\end{array}$ & $\begin{array}{c}\text { Lr46/Yr29 } \\
\text { (XWMC-44) }\end{array}$ & $\begin{array}{c}\text { Sr2/Yr30 } \\
(\mathrm{Xgwm}-533)\end{array}$ \\
\hline 70002 & $\begin{array}{c}\text { CHENAB2000/INQ.91/5/WBLL1 } * 2 / 4 / \text { SNI/TRAP } \\
\# 1 / 3 / \text { KAUZ } * 2 / \text { TRAP//KAUZ } \\
\text { PB-36259-0A-0A-0A-9A-0A } \\
\end{array}$ & + & - & + \\
\hline 70003 & $\begin{array}{c}\text { CHENAB2000/INQ.91/5/WBLL1*2/4/SNI/TRAP } \\
\# 1 / 3 / \text { KAUZ } * 2 / \text { TRAP//KAUZ } \\
\text { PB-36259-0A-0A-0A-12A-0A }\end{array}$ & + & + & + \\
\hline 70004 & $\begin{array}{c}\text { AS2002/5/FRET2 } * 2 / 4 / \mathrm{SNI} / \mathrm{TRAP} \# 1 / 3 / \mathrm{KAUZ} * 2 / \mathrm{T} \\
\text { RAP//KAL } \\
\text { PB-36109-0A-0A-0A-7A-0A }\end{array}$ & + & - & + \\
\hline 70007 & $\begin{array}{c}\text { FSD.08/6/BABAX/3/FASAN/Y//KAUZ/4/BABA } \\
\text { X/5/LU 26/HD2179 } \\
\text { PB-36121-0A-0A-0A-8A-0A }\end{array}$ & + & + & - \\
\hline 70012 & \begin{tabular}{|c} 
KAUZ//ALTAR84/AOS/3/MILAN/KAUZ/4/HUI \\
TES/5/KAUZ//ALTAR84/AOS/3/MILAN/KAUZ/ \\
4/HUITES \\
PB-36189-0A-0A-0A-5A-0A
\end{tabular} & + & - & + \\
\hline 70014 & $\begin{array}{c}\text { KAUZ//ALTAR84/AOS/3/MILAN/KAUZ/4/HUI } \\
\text { TES/5/KAUZ//ALTAR84/AOS/3/MILAN/KAUZ/ } \\
\text { 4/HUITES } \\
\text { PB-36189-0A-0A-0A-15A-0A }\end{array}$ & - & + & + \\
\hline 70015 & \begin{tabular}{|c|} 
KAUZ//ALTAR84/AOS/3/MILAN/KAUZ/4/HUI \\
TES/5/KAUZ//ALTAR84/AOS/3/MILAN/KAUZ/ \\
4/HUITES \\
PB-36189-0A-0A-0A-17A-0A
\end{tabular} & + & + & - \\
\hline 70025 & $\begin{array}{c}\text { OASIS/5*ANGRA//INQ.91///MILAN/S87230//BA } \\
\text { BAX } \\
\text { PB-36286-0A-0A-0A-8A-0A }\end{array}$ & + & - & + \\
\hline 70030 & $\begin{array}{c}\text { TRM//MAYA74'S'/MON'S'/3/INQ.91/4/PBW } \\
343 \\
\text { PB-36360-0A-0A-0A-11A-0A }\end{array}$ & + & - & + \\
\hline
\end{tabular}




\begin{tabular}{|c|c|c|c|c|}
\hline 70033 & $\begin{array}{c}\text { TRM//MAYA74'S'/MON'S'/3/INQ.91/4/PBW } \\
343 \\
\text { PB-36360-0A-0A-0A-19A-0A }\end{array}$ & - & + & + \\
\hline 70034 & $\begin{array}{c}\text { 87094/ERA//PAK- } \\
\text { 81/2*V87094/3/SHAFAQ06/4/MAYA/PVN } \\
\text { PB-36369-0A-0A-0A-11A-0A }\end{array}$ & + & + & + \\
\hline 70039 & $\begin{array}{c}\text { PFAU/MILAN/5/CHEN/A.SQ(TAUS)//BCN/3/VE } \\
\text { E\#7/BOW/4/PASTOR/6/QINGHAIBRI/WBLLI// } \\
\text { BRBT2 } \\
\text { PB-36377-0A-0A-0A-3A-0A }\end{array}$ & + & + & + \\
\hline 70043 & $\begin{array}{c}\text { INQALAB91 } * 2 / \text { KUKUNA//KIRITATI///V-09014 } \\
\text { PB-36447-0A-0A-0A-14A-0A }\end{array}$ & + & - & + \\
\hline 70046 & \begin{tabular}{|c|} 
AUQAB 2000*2/LAKTA-1 \\
PB.37077-0A-0A-0A-8A-0A
\end{tabular} & + & - & + \\
\hline 70047 & $\begin{array}{c}\text { AUQAB 2000*2/LAKTA-1 } \\
\text { PB.37077-0A-0A-0A-14A-0A }\end{array}$ & + & + & - \\
\hline 70048 & $\begin{array}{c}\text { AUQAB 2000*2/LAKTA-1 } \\
\text { PB.37077-0A-0A-0A-19A-0A }\end{array}$ & + & + & - \\
\hline 70050 & $\begin{array}{c}\text { FSD.08/6/BABAX/3/FASAN/Y//KAUZ/4/BABA } \\
\text { X/5/LU 26/HD2179/7/PB.96/87094//MH.97 } \\
\text { PB.37082-0A-0A-0A-9A-0A } \\
\end{array}$ & + & - & + \\
\hline 70054 & $\begin{array}{c}\text { FSD.08/6/BABAX/3/FASAN/Y//KAUZ/4/BABA } \\
\text { X/5/LU26/HD217 9/7/PB.96/87094//MH.97 } \\
\text { PB.37082-0A-0A-0A-19A-0A }\end{array}$ & + & + & + \\
\hline 70061 & \begin{tabular}{|c|} 
SH88/2*ATTILA/6/ACHTAR*3//KANZ/KS8585/ \\
4/MILAN/KAUZ//PRINIA/3/BAV92/5/MILAN/K \\
AUZ//PRINIA/3/BAV92 \\
PB No. 36821-0A-0A-0K-8A-0A
\end{tabular} & - & + & + \\
\hline 70064 & \begin{tabular}{|c|} 
CNDO/R143//ENTE/MEXI_2/3/AEGILOPSSQU \\
ARROSA(TAUS)/4/WEAVER/5/PICUS/6/TROS \\
T/7/TACUPETOF2001/8/OASIS/SKAUZ//4*BCN \\
/3/2*PASTOR \\
PB No. 36829-0A-0A-0K-15A-0A \\
\end{tabular} & + & + & + \\
\hline 70065 & \begin{tabular}{|c|} 
CNDO/R143//ENTE/MEXI_2/3/AEGILOPSSQU \\
ARROSA(TAUS)/4/WEAVER/5/PICUS/6/TROS \\
T/7/TACUPETOF2001/8/CROW'S'/NAC//BOW'S' \\
PB No. 36830-0A-0A-0K-1A-0A \\
\end{tabular} & + & - & + \\
\hline 70070 & \begin{tabular}{|c|} 
CNDO/R143//ENTE/MEXI_2/3/AEGILOPSSQU \\
ARROSA(TAUS)/4/WEAVER/5/PICUS/6/TROS \\
T/7/TACUPETOF2001/8/CROW'S'/NAC//BOW'S' \\
PB No. 36830-0A-0A-0K-12A-0A \\
\end{tabular} & + & + & + \\
\hline 70072 & \begin{tabular}{|c|} 
CNDO/R143//ENTE/MEXI_2/3/AEGILOPSSQU \\
ARROSA(TAUS)/4/WEAVER/5/PICUS/6/TROS \\
T/7/TACUPETOF2001/8/CROW'S'/NAC//BOW'S' \\
PB No. 36830-0A-0A-0K-14A-0A \\
\end{tabular} & + & - & + \\
\hline 70076 & $\begin{array}{c}\text { PFAU/SERI.1B//AMAD/3/INQALAB91*2/KUKU } \\
\text { NA/4/WBLL1 } 2 \text { 2/KURUKU/5/PVN/YACO/3/KA } \\
\text { UZ*2/TRAP// KAUZ } \\
\text { PB No. 36836-0A-0A-0K-10A-0A }\end{array}$ & + & + & + \\
\hline 70084 & $\begin{array}{l}\text { WHEAR/KRONSTADF2004//KAUZ/SITE } \\
\text { PB No. 36880-0A-0A-0K-1A-0A }\end{array}$ & + & - & + \\
\hline 70085 & $\begin{array}{l}\text { WHEAR/KRONSTADF2004//KAUZ/SITE } \\
\text { PB No. 36880-0A-0A-0K-11A-0A }\end{array}$ & + & + & + \\
\hline
\end{tabular}




\begin{tabular}{|c|c|c|c|c|}
\hline 70086 & $\begin{array}{l}\text { WHEAR/KRONSTADF2004//KAUZ/SITE } \\
\text { PB No. 36880-0A-0A-0K-12A-0A } \\
\end{array}$ & + & - & + \\
\hline 70087 & $\begin{array}{l}\text { WHEAR/KRONSTADF2004//KAUZ/SITE } \\
\text { PB No. 36880-0A-0A-0K-13A-0A }\end{array}$ & + & + & - \\
\hline 70088 & $\begin{array}{l}\text { WHEAR/KRONSTADF2004//KAUZ/SITE } \\
\text { PB No. 36880-0A-0A-0K-15A-0A }\end{array}$ & - & + & + \\
\hline 70092 & $\begin{array}{l}\text { PFAU/MILAN//PBW343*2/TUKURU/3/NR381 } \\
\text { PB No. 36885-0A-0A-0K-13A-0A }\end{array}$ & + & - & + \\
\hline 70096 & $\begin{array}{c}\text { CROC_1/AE.SQUARROSA(205)//KAUZ/3/PAST } \\
\text { OR/4/THELIN/5/INQ/AUQAB } \\
\text { PB No. 36893-0A-0A-0K-3A-0A } \\
\end{array}$ & - & + & + \\
\hline 70098 & $\begin{array}{c}\text { CROC_1/AE.SQUARROSA(205)//KAUZ/3/PAST } \\
\text { OR/4/THELIN/5/INQ/AUQAB } \\
\text { PB No. 36893-0A-0A-0K-5A-0A } \\
\end{array}$ & + & - & + \\
\hline 70101 & $\begin{array}{c}\text { MINO/898.97/4/INIA66/7C//MAYA/3/PCI/TRM } \\
\text { PB No. 36894-0A-0A-0K-15A-0A }\end{array}$ & + & - & + \\
\hline 70103 & \begin{tabular}{|c|} 
CNDO/R143//ENTE/MEXI_2/3/AEGILOPSSQU \\
ARROSA(TAUS)/4/WEAVER/5/IRENA/6/LERK \\
E/7/TAN/PEW//SARA/3/CBRD \\
PB No. 36976-0A-0A-0K-4A-0A \\
\end{tabular} & + & + & + \\
\hline 70104 & \begin{tabular}{|c|} 
CNDO/R143//ENTE/MEXI_2/3/AEGILOPSSQU \\
ARROSA(TAUS)/4/WEAVER/5/IRENA/6/LERK \\
E/7/TAN/PEW//SARA/3/CBRD \\
PB No. 36976-0A-0A-0K-10A-0A \\
\end{tabular} & + & + & + \\
\hline 70107 & \begin{tabular}{|c|} 
CNDO/R143//ENTE/MEXI_2/3/AEGILOPSSQU \\
ARROSA(TAUS)/4/WEAVER/5/IRENA/6/LERK \\
E/7/TAN/PEW//SARA/3/CBRD \\
PB No. 36976-0A-0A-0K-18A-0A \\
\end{tabular} & + & + & - \\
\hline 70108 & \begin{tabular}{|c|} 
PBW343*2/KUKUNA//KRONSTADF2004/3/PB \\
W343*2/KUKUNA/4/CHENAB2000/INQ.91 \\
PB No. 36978-0A-0A-0K-8A-0A \\
\end{tabular} & + & + & - \\
\hline
\end{tabular}

+ sign shows the presence of rust resistance genes in wheat genotypes while

- sign shows absence of rust resistance genes in wheat genotypes

SSR marker Xwmc-44 exhibited linkage to $L r 46 / Y r 29$ leaf and stripe rust resistance gene located on chromosome arm 7B. Its bands showed the amplification in the range of 242 bp. Eight elite lines were resistant while 16 advanced lines like V-70011, V-70012, V-70013, V-70014, V-70016, V-70017, V-70018, V-70019, V-70020, V-70021, V70022, V-70023, V-70024, V-70026, V-70027 and V-70028 were found susceptible with $L r 46 / Y r 29$ and the amplification of only 24 elite lines by polymerase chain reaction has been demonstrated (Fig. 2). PCR-based diagnostic marker XGWM-533 was linked to $\operatorname{Sr} 2 / \mathrm{Yr} 30$ stem and stripe rust resistance gene. $\operatorname{Sr} 2 / \mathrm{Yr} 30$ was exist on chromosomal loci 3BS. All advanced lines indicated the presence of this gene with the band size of $120 \mathrm{bp}$. Twenty four lines amplified by PCR showed that 13 lines were resistant while 11 genotypes i.e. V-70007, V-70015, V-70029, V-70031, V-70032, V-70035, V-70036, V-70037, V-70038, V-70040 and V-70041 were found susceptible (Fig. 3).

From this investigation it was concluded that among 112 advanced lines, only 10 lines V-70003, V-70034, V-70039, V-70054, V-70064, V-70070, V-70076, V-70085, V-70103 and V-70104 demonstrated the association of 3 designated slow rusting/race non-specific genes. This is very significant linkage, as it gives resistance against all 3 types of rust i.e. stripe, leaf and stem rust. Similarly, 15 genotypes Viz. V-70002, V- 
70004, V-70012, V-70025, V-70030, V-70043, V-70046, V-70050, V-70065, V-70072, V-70084, V-70086, V-70092, V-70098 and V-70101 exhibited the linkage of Lr34/Yr18 and $S r 2 / Y r 30$. Linkage of $L r 46 / Y r 29$ and $S r 2 / Y r 30$ was indicated in 5 lines viz. V70014, V-70033, V-70061, V-70088, V-70096 and the association of Lr34/Yr18 and Lr46/Yr29 was identified in 7 lines including V-7007, V-70015, V-70047, V-70048, V70087, V-70107, and V-70108. All these brilliant advanced lines having durable type resistance along with low values of area under disease progress curve may be used in future hybridization schemes to enhance level of resistance in the adapted wheat cultivars of Pakistan (Inqilab-91, Uqab-2000, AS-2002, Seher-2006 and Fareed-06 etc).

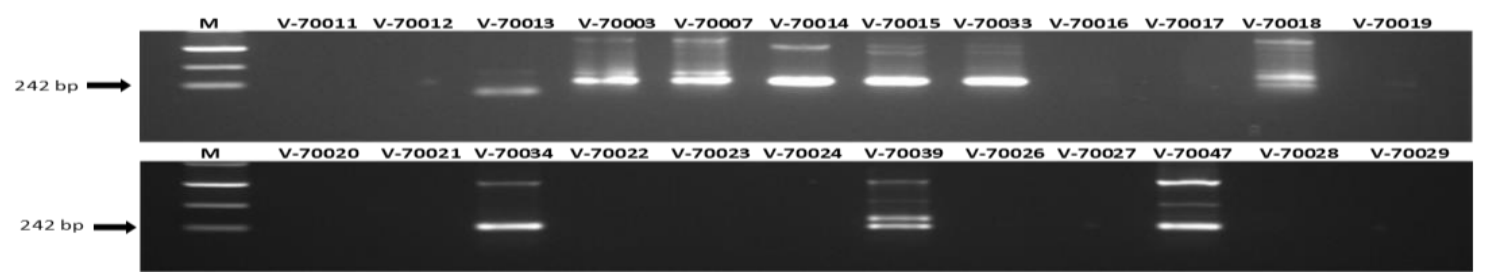

Figure 2. PCR amplification profile of 24 advanced lines for SSR marker XWMC-44 linked to Lr46/Yr29; $M=1 \mathrm{~Kb}$ DNA Ladder Marker

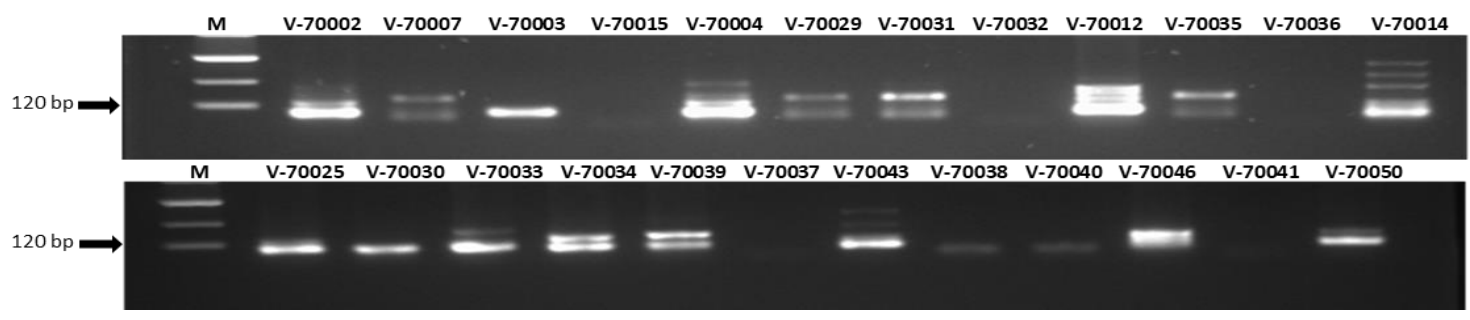

Figure 3. PCR amplification profile of 24 advanced lines for SSR marker XGWM-533 linked to Sr2/Yr30; $M=1 \mathrm{~Kb}$ DNA Ladder Marker

\section{Yield testing of selected wheat elite lines on the basis of their genetic traits}

Basic statistics for all parameter is described in Table 6 showed a considerable variability among germplasm that were under study. Medium to high variance was determined for plant height $(\mathrm{cm})$, thousand grain weight, number of spikelet per spike and grain yield $\left(\mathrm{kg} \mathrm{ha}^{-1}\right)$ while small variance was determined for spike length $(\mathrm{cm})$ and protein percentage.

\section{Correlation analyses}

A matrix of correlation coefficient among grain yield and its component was determined (Table 7). Results indicated that plant height exhibited significant correlation with protein $(\%)$ while highly significant correlation with spike length. Thousand grain weight exhibited significant relationship with number of spikelet per spike. A highly significant correlation was observed between grain yield $\left(\mathrm{kg} / \mathrm{ha}^{-1}\right)$ and 1000 grain weight indicating the need of more emphasis on these parameters to increase yield in wheat. 
Table 6. Basic statistics for 6 quantitative variables of 112 advance lines along with five checks

\begin{tabular}{c|c|c|c|c|c}
\hline Sr. no & Parameters & Mean \pm S.D. & Minimum value & Maximum value & Variance \\
\hline 1. & $\mathrm{PH}(\mathrm{cm})^{\mathrm{a}}$ & $106.479 \pm 7.1192$ & 86.000 & 124.000 & 50.70 \\
2. & $\mathrm{GY}\left(\mathrm{Kg} / \mathrm{ha}^{-1}\right)^{\mathrm{b}}$ & $3762.829 \pm 609.2895$ & 2469.000 & 4800.000 & 371233.7 \\
3. & $\mathrm{P}(\%)^{\mathrm{c}}$ & $11.430 \pm 0.8742$ & 9.400 & 13.600 & 0.8 \\
4. & $\mathrm{TGW}(\mathrm{g})^{\mathrm{d}}$ & $36.921 \pm 3.5343$ & 30.000 & 45.000 & 12.5 \\
5. & $\mathrm{SL}(\mathrm{cm})^{\mathrm{e}}$ & $9.392 \pm 1.1790$ & 6.980 & 12.920 & 1.4 \\
6. & $\mathrm{SSP}^{\mathrm{f}}$ & $45.458 \pm 4.8649$ & 36.430 & 55.980 & 23.7 \\
\hline
\end{tabular}

aPlant height $(\mathrm{cm}) ;{ }^{\mathrm{b}}$ Grain yield $\left(\mathrm{Kg} \mathrm{ha}^{-1}\right)$; ${ }^{\mathrm{c}}$ Protein $(\%) ;{ }^{\mathrm{d}} 1000$ grain weight $(\mathrm{g})$; ${ }^{\mathrm{S}}$ Spike length $(\mathrm{cm})$;

${ }^{\mathrm{f}}$ Number of spikelet per spike

Table 7. Correlation coefficient ( $r$ ) matrix for estimated six parameters of genotypes

\begin{tabular}{|c|c|c|c|c|c|c|}
\hline Parameters & $\mathrm{X} 1$ & $\mathrm{X} 2$ & $\mathrm{X3}$ & $\mathrm{X} 4$ & $\mathrm{X5}$ & $\mathrm{X6}$ \\
\hline $\mathrm{PH}(\mathrm{cm})(\mathrm{X} 1)$ & 1.000 & & & & & \\
\hline GY $\left(\mathrm{kg} / \mathrm{ha}^{-1}\right)(\mathrm{X} 2)$ & $\begin{array}{l}0.077 \\
0.406\end{array}$ & 1.000 & & & & \\
\hline $\mathrm{P}(\%)(\mathrm{X} 3)$ & $\begin{array}{c}0.193 * \\
0.037\end{array}$ & $\begin{array}{l}0.152 \\
0.102\end{array}$ & 1.000 & & & \\
\hline TGW (g) (X4) & $\begin{array}{l}0.044 \\
0.638 \\
\end{array}$ & $\begin{array}{c}0.252 * * \\
0.006 \\
\end{array}$ & $\begin{array}{l}0.062 \\
0.504 \\
\end{array}$ & 1.000 & & \\
\hline $\mathrm{SL}(\mathrm{cm})(\mathrm{X} 5)$ & $\begin{array}{c}0.256^{* * *} \\
0.005\end{array}$ & $\begin{array}{l}0.074 \\
0.429\end{array}$ & $\begin{array}{c}0.226^{*} \\
0.014\end{array}$ & $\begin{array}{l}0.170 \\
0.067\end{array}$ & 1.000 & \\
\hline SSP (X6) & $\begin{array}{l}0.098 \\
0.296\end{array}$ & $\begin{array}{l}0.079 \\
0.396\end{array}$ & $\begin{array}{l}0.054 \\
0.565\end{array}$ & $\begin{array}{c}0.205^{*} \\
0.026\end{array}$ & $\begin{array}{c}0.482 * * \\
0.000\end{array}$ & 1.000 \\
\hline
\end{tabular}

Upper values indicated Pearson's correlation coefficient. Lower values indicated level of significance at $5 \%$ probability. $*=$ significant $(\mathrm{P}<0.05) ; * *$ highly significant $(\mathrm{P}<0.01)$. Abbreviations as in Table 6

Cluster analysis categorized 112 wheat lines along with five checks into 5 clusters (Table 8; Fig. 4). Distribution pattern of all the genotypes into various clusters exhibited the presence of considerable genetic variability among the genotypes for most of the traits studied. Association among these cluster members showed that clusters V, IV and II showed maximum, while cluster I and III indicated minimum mean values for most of the traits respectively (Table 9). Results confirmed that all genotypes formed in cluster $\mathrm{V}$ under trial condition exhibited highest mean values for all traits. After testing under different environmental conditions, all 13 lines of cluster V except V-70078 due to lack of resistance genes (Table 5) could be used for their direct release as variety. Furthermore, all these outstanding lines might be used in hybridization programs to develop rust resistance and high yield varieties.

Six PCs (PC1-PC6) were made from original statistical data revealing 98\% of total variation (Table 10). Out of six principal components three PCs (PC1-PC3) have Eigen value greater than 1 , accounted for individual variance values of $30.93,18.44$, and $17.84 \%$ with $67.21 \%$ of cumulative variation of grain yield respectively. The first two PCs were plotted on PC axis 1 and 2 that showed high variability in the existing wheat lines and checks (Fig. 5). Traits with largest absolute values closer to unity within the 
PC1 influence the clustering more than those with lower absolute values closer to zero (Chahal and Gosal, 2002). Therefore, in this investigation, differentiation of the advanced lines into different cluster was due to the cumulative effect of a number of traits rather than the contribution of specific few characters. All traits in PC1 showed negative component value whereas, grain yield $\left(\mathrm{kg} / \mathrm{ha}^{-1}\right)$ exhibited great effect in second Principal Component (PC2). Traits having relatively higher value in the PC3 like number of spikelet per spike, thousand grain weight and spike length had more contribution to the total variation and they were the ones that most differentiated the clusters. Plant height $(\mathrm{cm})$, grain yield $\left(\mathrm{kg} / \mathrm{ha}^{-1}\right)$ thousand grain weight $(\mathrm{g})$ in the PC4, plant height $(\mathrm{cm})$, protein $(\%)$, thousand grain weight $(\mathrm{g})$ in PC5, spike length $(\mathrm{cm})$, grain yield $\left(\mathrm{kg} / \mathrm{ha}^{-1}\right)$ and thousand grain weight $(\mathrm{g})$ in PC5 were the major contributors to each Principal Components (PC). The current investigation confirmed that advanced wheat lines exhibited wide range of variations for the traits studies and it also proposed that ample prospects for genetic improvement of wheat genotypes through selection directly from bread wheat genotypes and conservation of the germplasm for future utilization.

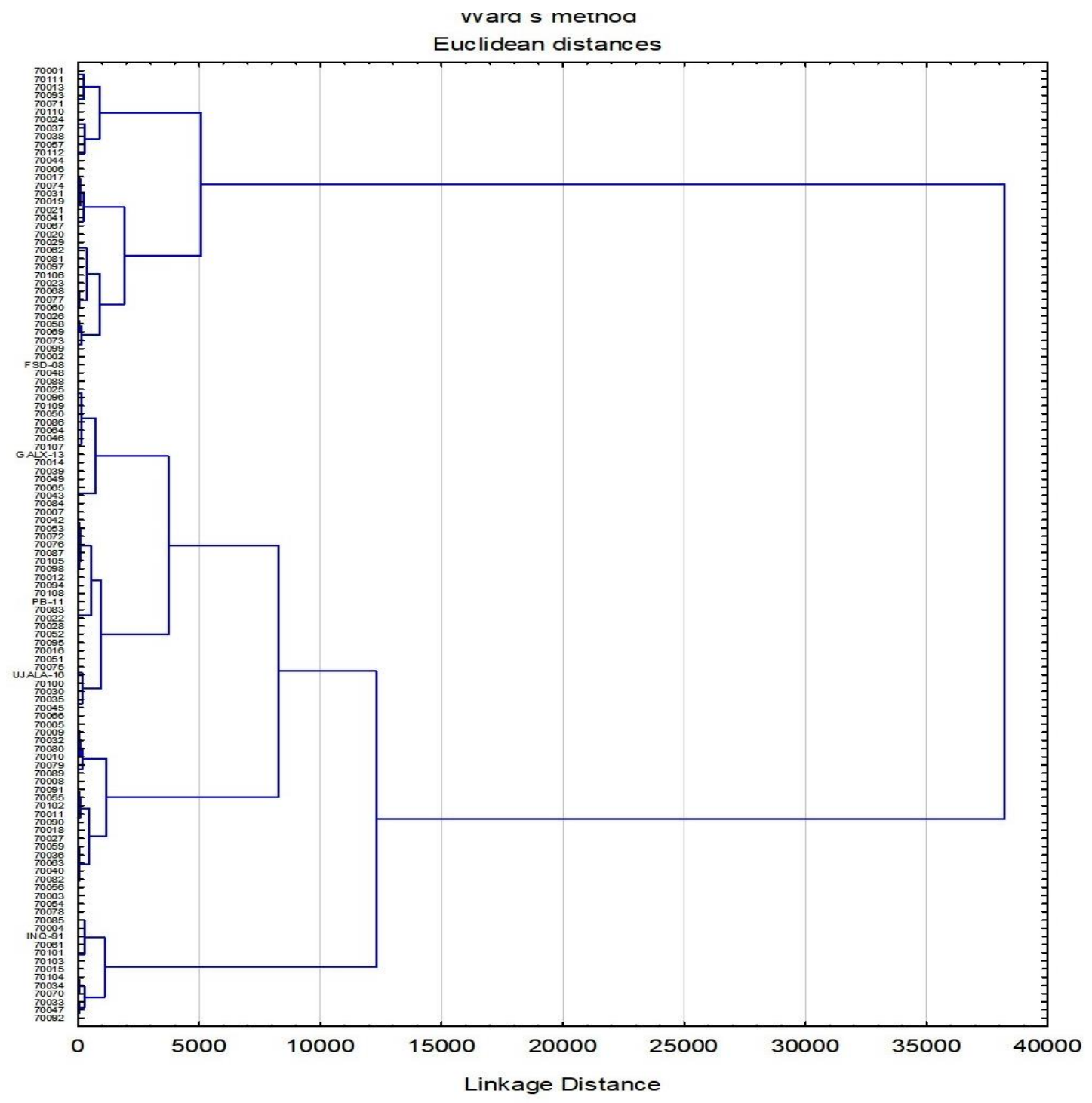

Figure 4. Cluster diagram of 112 advance lines and varieties based on sic traits under study 


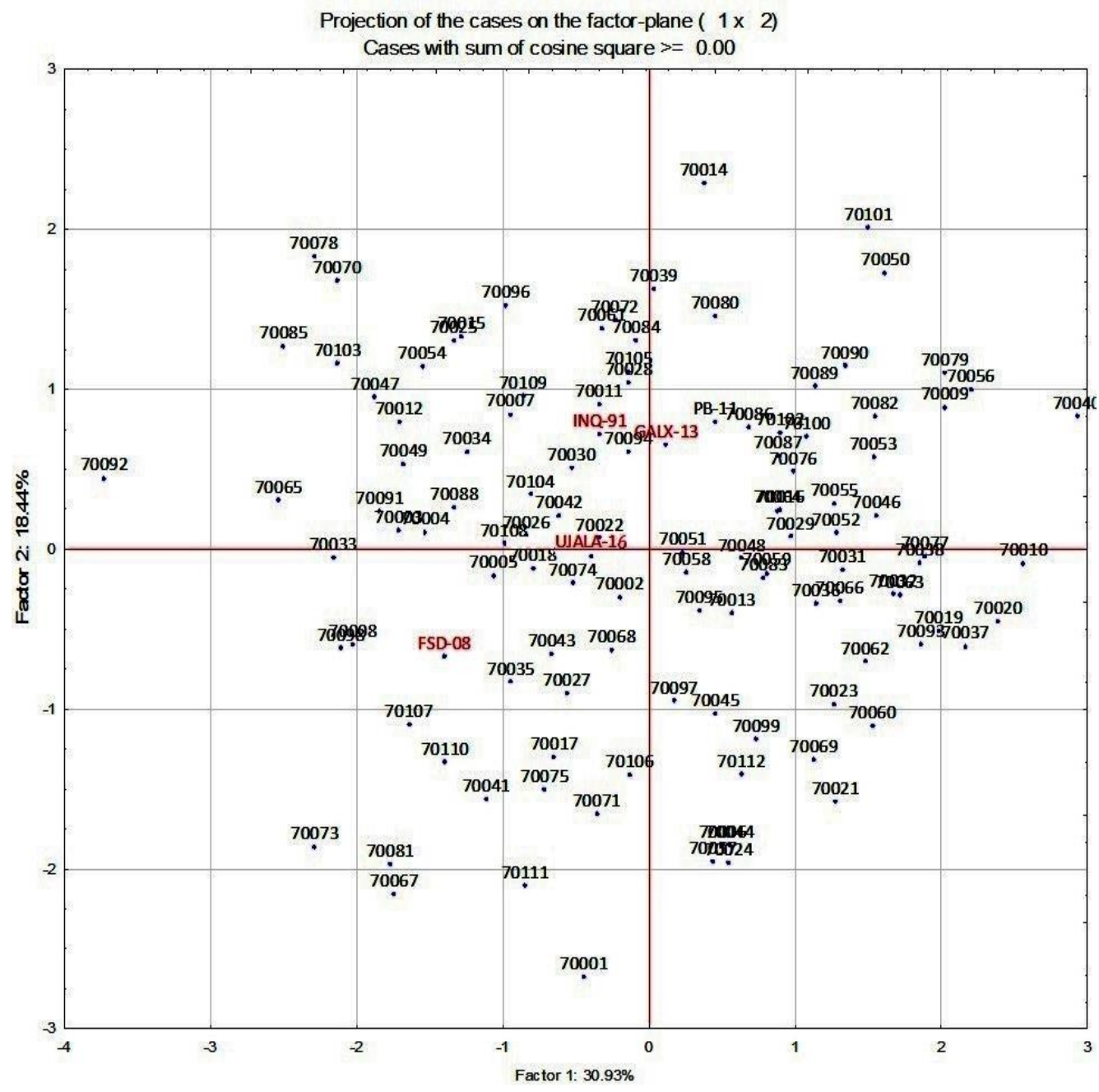

Figure 5. Scattered diagram of first two PCs (Factor 1 as PC1 and Factor 2 as PC2)

\section{Discussion}

PCR-based DNA markers associated with genes controlling target economic traits have significant role to attain sustained wheat production. Molecular marker-trait combinations give an effective alternative to phenotyping for selecting varieties that have linkage of desirable genes in breeding populations.

Here we used three SSR markers XGWM-533, XWMC-44 and X-barc-352 for effective marker assisted selection of Sr2/Yr30, Lr46/Yr29 and Lr34/Yr18 in selected wheat elite lines. This investigation exhibited, among all tested genotypes only 10 advanced lines showed a tight linkage to Sr2/Yr30, Lr46/Yr29 and Lr34/Yr18 having durable type resistance under the severe disease epidemics. According to Singh and Bowden (2011), resistance near immunity can be achieved by combining 4-5 race nonspecific resistance genes in a cultivar. Though, slow level of resistance can be attained by combining 2 to 3 race non-specific/minor genes in a genotype (Lagudah et al., 2009). International Maize and Wheat Improvement Centre (CIMMYT) and Ayub Agriculture Research Institute (AARI) planned a technique of combining race non-specific resistance genes alone or in linkage with some other genes to control recently evolved strains of wheat rust (Rehman et al., 2013). 
Table 8. Five cluster grouping wheat lines based on six parameters under study

\begin{tabular}{|c|c|c|}
\hline Cluster & Frequency & Cluster membership \\
\hline I & 22 & $\begin{array}{c}\text { V-70005, V-70009, V-70010, V-70011, V-70018, V-70026, V-70027, V-70032, } \\
\text { V-70036, V-70040, V-70056, V-70058, V-70059, V-70063, V-70069, V-70073, } \\
\text { V-70079, V-70080, V-70082, V-70089, V-70090 and V-70099 }\end{array}$ \\
\hline II & 29 & \begin{tabular}{|} 
V-70007, V-70008, V-70012, V-70016, V-70022, V-70028, V-70030, V-70035, \\
V-70042, V-70045, V-70051, V-70052, V-70053, V-70055, V-70066, V-70072, \\
V-70075, V-70076, V-70083, V-70087, V-70091, V-70094, V-70095, V-70100, \\
V-70102, V-70105, V-70108, UJALA-16 and PB-11
\end{tabular} \\
\hline III & 30 & \begin{tabular}{|c} 
V-70001, V-70006, V-70013, V-70017, V-70019, V-70020, V-70021, V-70023, \\
V-70024, V-70029, V-70031, V-70037, V-70038, V-70041, V-70044, V-7005, \\
V-70060, V-70062, V-70067, V-70068, V-70071, V-70074, V-70077, V-70081, \\
V-70093, V-70097, V-70106, V-70110, V-70111 and V-70112
\end{tabular} \\
\hline IV & 21 & $\begin{array}{c}\text { V-70002, V-70014, V-70025, V-70033, V-70039, V-70043, V-70046, V-70048, } \\
\text { V-70049, V-70050, V-70064, V-70065, V-70084, V-70086, V-70088, V-70096, } \\
\text { V-70098, V-70107, V-70109, GALX-13 and FSD-08 }\end{array}$ \\
\hline $\mathrm{V}$ & 15 & $\begin{array}{c}\text { V-70003, V-70004, V-70015, V-70034, V-70047, V-70054, V-70061, V-70070, } \\
\text { V-70078, V-70085, V-70092, V-70101, V-70103, V-70104 and INQ-91 }\end{array}$ \\
\hline
\end{tabular}

Table 9. Mean and standard deviation for five clusters based on six parameters under study

\begin{tabular}{c|c|c|c|c|c}
\hline \multirow{2}{*}{ Traits } & \multicolumn{5}{|c}{ Mean \pm SD } \\
\cline { 2 - 6 } & Cluster - I & Cluster - II & Cluster - III & Cluster - IV & Cluster - V \\
\hline PH (cm) & $104.55 \pm 9.64$ & $106.41 \pm 5.17$ & $106.33 \pm 7.88$ & $106.81 \pm 6.49$ & $109.27 \pm 4.92$ \\
GY (g) & $3583.3 \pm 148.9$ & $3969.0 \pm 92.0$ & $2911.2 \pm 235.0$ & $4246.7 \pm 72.8$ & $4653.5 \pm 100.0$ \\
P (\%) & $11.21 \pm 0.96$ & $11.39 \pm 0.76$ & $11.40 \pm 0.90$ & $11.34 \pm 0.96$ & $12.00 \pm 0.59$ \\
TGW (g) & $36.62 \pm 3.81$ & $36.62 \pm 3.46$ & $35.80 \pm 2.64$ & $37.48 \pm 3.83$ & $39.41 \pm 3.51$ \\
SL (cm) & $8.73 \pm 1.10$ & $9.29 \pm 0.88$ & $9.50 \pm 1.41$ & $9.69 \pm 1.02$ & $9.93 \pm 1.16$ \\
SSP & $43.46 \pm 4.55$ & $45.18 \pm 4.72$ & $45.55 \pm 4.96$ & $46.58 \pm 5.09$ & $47.18 \pm 4.58$ \\
\hline
\end{tabular}

Abbreviations as in Table 6

Table 10. Eigen values, percent individual variance and percent cumulative variance for 6 characters studied on 112 advanced wheat lines along with five checks

\begin{tabular}{c|c|c|c|c|c|c}
\hline Parameters & PC1 & PC2 & PC3 & PC4 & PC5 & PC6 \\
\hline PH $(\mathbf{c m})$ & -0.47 & -0.33 & -0.47 & 0.65 & 0.04 & -0.12 \\
GY $\left(\mathbf{k g ~ h a}^{-\mathbf{1}}\right)$ & -0.40 & 0.71 & -0.25 & 0.047 & -0.53 & 0.05 \\
P $(\%)$ & -0.46 & -0.06 & -0.64 & -0.56 & 0.21 & -0.14 \\
TGW (g) & -0.49 & 0.59 & 0.24 & 0.16 & 0.57 & 0.01 \\
SL (cm) & -0.77 & -0.34 & 0.18 & -0.10 & -0.06 & 0.49 \\
SSP & -0.67 & -0.19 & 0.53 & -0.11 & -0.19 & -0.43 \\
Eigen value & 1.85 & 1.11 & 1.07 & 0.79 & 0.70 & 0.47 \\
Individual variance (\%) & 30.93 & 18.44 & 17.84 & 13.25 & 11.68 & 7.85 \\
Cumulative variance (\%) & 30.93 & 49.37 & 67.21 & 80.46 & 92.14 & 98.00 \\
\hline
\end{tabular}

Abbreviations as in Table 6 
These genotypes are important source of rust protection with high yield potential. The resistance in determined genotypes seems to be durable in nature. The race specific resistance controlled by the parent lines was vulnerable as the single line V-70001 exhibited severe disease outbreaks ranging from 50-60\% in the disease screening plots. Combination form these parent lines against common stripe and leaf rust races proved very effective with lower disease severity in the country (Hussain et al., 2006).

Many new released cultivars have been banned for general cultivation only because of disease vulnerability against novel stripe and leaf rust races (Khan et al., 2002). Combining 2-3 or more genes in a wheat genotype for race non-specific resistance has remained the main emphasis of researchers to combat the changing nature of novel virulent races (Roelfs, 1988). To contest this problem, DNA molecular marker technique was used for improving rust resistance through combining various race nonspecific resistance genes in selected wheat lines. Genotypes possessing slow rust linkage illustrated lower area under disease progress curve at adult-plant stage have durable resistance as also indicated by various researchers (Bariana et al., 2001; Singh et al., 2005; Singh and Bowden, 2011). Because the race non-specific resistance like partial and durable rust resistance is polygenic as observed elsewhere, therefore, it remains effective for longer time period, even if the pathogen change its virulence pattern through mutation or recombination (Dehghani and Moghaddam, 2004). Thus, in present study, genotypes having low rust intensity could be considered as durable lines carrying high level of rust resistance to $S r 2, \operatorname{Lr} 46$, and $\operatorname{Lr} 34$ virulences, that might be used in future hybridization schemes to protect crop stability. For its relative ease, productivity and specificity, many researchers have examined the robustness of these molecular markers to identify the occurrence of stripe and leaf rust resistance in wheat germplasm (Dakouri et al., 2013; Lagudah et al., 2006; Mustafa et al., 2013).

In order to evaluate, maintain and use advanced lines effectively it is necessary to study the extent of genetic variability available. Morphological characterization has been successfully used for determination of genetic variability and variety development (Fufa et al., 2005). Analysis of genetic variability through cluster and PCA analyses among germplasm collection help in sorting and core collection of genotype that used for specific breeding purpose (Muhammadi and Prasana, 2003). The cluster analysis classified lines into clusters that showed high intra cluster homogeneity and inter cluster heterogeneity (Jaynes et al., 2003). Considering the significant correlation between grain yield and thousand grain weight and also that the average values of these two parameters for Cluster $\mathrm{V}$ are greater than the average of all elite lines. Member of this cluster may be used to increase yield in breeding schemes. The results of this investigation are in line with the findings of Leilah and Al-Khateeb (2005), Ali et al. (2008), Hendawy et al. (2011), and Hristov et al. (2011) who demonstrated the significant correlation between grain yield and other quantitative variables. Spike length and plant height were positively correlated. Same was observed in case of thousand grain weight and number of spikelet per spike i.e. as it decreased the grain yield $\left(\mathrm{kg} \mathrm{ha}^{-}\right.$ ${ }^{1}$ ) also decrease and as it increased the grain yield also increase (Kamyab et al., 2009).

The principal component analysis showed that, all six PCs had $98 \%$ of total variation in the data (Hailegiorgis et al., 2011). Principal component and cluster analyses allowed natural clustering of wheat germplasm. Accordingly, the various measurement methods can be properly used for clustering of wheat germplasm (Kraic et al., 2009). Thus results demonstrated that PCA based cluster analyses is more precise indicator of difference among wheat advance lines than cluster analyses not based on PCA. 
However, increased yield potential is stated goal for researchers. Progress in yield characteristics results from the progressive accumulation of minor genes possessing high yield potential (Ajmal et al., 2013). In present investigation, 32 lines showed the linkage of $L r 34 / Y r 18,22$ lines demonstrated $L r 46 / Y r 29$ and 30 lines exhibited the linkage of $S r 2 / Y r 30$. Determining the existence of $S r 2, L r 46$, and $\operatorname{Lr} 34$ genes in current elite lines is useful to predict field response. The stability of these selected elite lines helps decision in selecting parentage for future hybridization and to develop new varieties with improved yellow and leaf rust resistance. Thus, the scheme of combining race non-specific genes through breeding is the best way to attain durable resistance in wheat germplasm under continuously changing virulence pattern in the country.

\section{Conclusion}

The advanced lines V-70003, V-70034, V-70039, V-70054, V-70064, V-70070, V70076, V-70085, V-70103 and V-70104 exhibited the combination of all three slow rusting genes ( $\mathrm{Lr34/Yr18,Lr46/Yr29}$ and $\mathrm{Sr} 2 / \mathrm{Yr} 30)$. In principal component analysis was demonstrated that six principal components PC1, PC2, PC3, PC4, PC5 and PC6 accounted for $98.00 \%$ of the total variation. The first two principal components PC1 and PC2 with values of 30.93 and $18.44 \%$, respectively, contributed more to the total variation indicating hybridization breeding program can be initiated by the selection of genotypes from the PC1 and PC2. Among all traits evaluated plant height $(\mathrm{cm})$, Plant height $(\mathrm{cm})$, grain yield $\left(\mathrm{kg} \mathrm{ha}^{-1}\right)$, protein $(\%)$, thousand grain weight $(\mathrm{g})$, spike length $(\mathrm{cm})$, and number of spikelet per spike in each principal component contributed more to the total genetic variations. In conclusion, the crosses between advanced lines selected from cluster- $\mathrm{V}$ are expected to produce better genetic recombination and segregation in their progenies. Therefore, these advanced lines need to be crossed and selected to develop high yielding pure line variety.

Acknowledgements. The authors dedicated this research manuscript to Dr. Makhdoom Hussain, Director Wheat Research Institute AARI, Faisalabad for providing research facilities at Wheat Research Experimental Area, Faisalabad.

\section{REFERENCES}

[1] Ahmad, J. N., Ahmad, S. J., Aslam, M., Ahmad, M. A., Contaldo, N., Paltrinieri, S., Bertaccini, A. (2017): Molecular and biologic characterization of a phytoplasma associated with Brassica campestris phyllody disease in Punjab province, Pakistan. European Journal of Plant Pathology 149(1): 117-125.

[2] Ajmal, S., Minhasi, N. M., Hamdani, A., Shakiri, A., Zubair, M., Ahmad, Z. (2013): Multivariate analysis of genetic divergence in wheat (Triticum aestivum) germplasm. Pakistan Journal of Botany 45(5): 1643-1648.

[3] Ali, Y., Atta, B. M., Akhter, J., Monneveux, P., Lateef, Z. (2008): Genetic variability, association and diversity studies in wheat (Triticum aestivium L.) germplasm. - Pakistan Journal of Botany 40(5): 2087-2097.

[4] Bansal, U. K., Kazi, A. G., Singh, B., Hare, R. A., Bariana, H. S. (2014): Mapping of durable stripe rust resistance in a durum wheat cultivar Wollaroi. - Molecular Breeding 33: 51-59. 
[5] Baranwal, D. K., Mishra, V. K., Vishwakarma, M. K., Yadav, P. S., Arun, B. (2012): Studies on genetic variability, correlation and path analysis for yield and yield contributing traits in wheat (T. aestivum L. em Thell.). - Plant Arch 12(1): 99-104.

[6] Bariana, H. S., Hayden, M. J., Ahmed, N. U., Bell, J. A., Sharp, P. J., McIntosh, R. A. (2001): Mapping of durable adult plant and seedling resistances to stripe rust and stem rust diseases in wheat. - Australian Journal of Agricultural Research 52: 1247-1255.

[7] Borner, A., Chebotar, S., Korzun, V. (2000): Molecular characterization of the genetic integrity of wheat (Triticum aestivum L.) germplasm after long-term maintenance. Theoretical and Applied Genetics 100: 494-497.

[8] Burkhamer, R. L., Lanning, S. P., Martens, R. J., Martin, J. M., Talbert, L. E. (1998): Predicting progeny variance from parental divergence in hard red spring wheat. - Crop Science 38: 243-248.

[9] Chahal, G. S., Gosal, S. S. (2002): Principles and Procedures of Plant Breeding: Biotechnology and Conventional Approaches. - Alpha Science International, Oxford, UK.

[10] Chen, H. B., Martin, J. M., Lavin, M., Talbert, L. E. (1994): Genetic diversity in hard red spring wheat based on sequence-tagged-site PCR markers. - Crop Science 34: 162-1632.

[11] Cheng, P., Chen X. M. (2014): Virulence and molecular analyses support asexual reproduction of Puccinia striiformis f. sp. tritici in the U. S. Pacific Northwest. Phytopathology 104: 1208-1220.

[12] Dakouri, A., McCallum, B. D., Radovanovic, N., Cloutier, S. (2013): Molecular and phenotypic characterization of seedling and adult plant leaf rust resistance in a world wheat collection. - Molecular Breeding 32: 663-677.

[13] Dehghani, H., Moghaddam, M. (2004): Genetic analysis of the latent period of stripe rust in wheat seedlings. - Journal of Phytopathology 152: 325-330.

[14] Devos, K. M., Gale, M. D. (1992): The use of random amplified polymorphic DNA markers in wheat. - Theoretical and Applied Genetics 84: 567-572.

[15] Fahima, T., Röder, M. S., Wendehake, K., Kirzhner, V. M., Nevo, E. (2002): Microsatellite polymorphism in natural populations of wild emmer wheat, Triticum dicoccoides, in Israel. - Theoretical and Applied Genetics 104: 17-29.

[16] FAO (2016): What is Conservation Agriculture? - http://www.fao.org/ag/ca/la.htm 1. FAO, Rome.

[17] Fufa, H., Baenizger, P. S., Beecher, B. S., Dweikat, I., Graybosch, R., Eskridge, A. K. M. (2005): Comparison of phenotypic and molecular-based classifications of hard red winter wheat cultivars. - Euphytica 145: 133-146.

[18] Hailegiorgis, D., Mesfin, M., Genet, T. (2011): Genetic Divergence Analysis on Some Bread Wheat Genotypes Grown in Ethiopia. - Journal of Central European Agriculture 12: 344-352.

[19] Hammer, K., Filatenko, A. A., Korzun, V. (2000): Microsatellite markers: a new tool for distinguishing diploid wheat species. - Genetic Resources and Crop Evolution 47: 497505 .

[20] Hendawy, S. E., Sakagami, J. I., Hu, Y., Schmidhalter, U. (2011): Screening Egyptian wheat genotypes for salt tolerance at early growth stages. - International Journal of Plant Breeding 5(3): 1735-8043.

[21] Hovmoller, M. S., Amor, H., Yahyaoui, E. A., Annemarie, F. J. (2008): Rapid global spread two aggressive strains of a wheat rust fungus. - Molecular Ecology 17(17): 38183826.

[22] Hristov, N., Mladenov, N., Spika, A. K., Jeromela, A. M., Jockovic, B., Jacimovic, G. (2011): Effect of environment and genetic factors on the correlation and stability of grain yield components in wheat. - Genetika 43(1): 141-152.

[23] Huang, X. Q., Hsam, S. L. K., Zeller, F. J., Wenzel, G., Mohler, V. (2000): Molecular mapping of the wheat powdery mildew resistance gene Pm24 and marker validation for molecular breeding. - Theoretical and Applied Genetics 101: 407-414. 
[24] Hussain, M., Ayub, N., Khan, S. M., Khan, M. A., Muhammad, F., Hussain, M. (2006): Pyramiding rust resistance and high yield in bread wheat. - Pakistan Journal of Phytopathology 18: 11-21.

[25] Hussain, M., Khan, M. A., Hussain, M., Javed, N., Khaliq, I. (2015): Application of phenotypic and molecular markers to combine genes for durable resistance against rust virulences and high yield potential in wheat. - Internal Journal Agriculture and Biology 17: 421-430.

[26] Jaynes, D. B., Kaspar, T. C., Colvin, T. S., James, D. E. (2003): Cluster analysis of spatiotemporal corn yield pattern in an Iowa field. - Agronomy Journal 95: 574-586.

[27] Joshi, A. K., Kumar, S., Chand, R., Ortiz-Ferrara, G. (2004): Inheritance of resistance to spot blotch caused by Bipolaris sorokiniana in spring wheat. - Plant Breeding 123: 213-219.

[28] Joshi, C. P., Nguyen, H. T. (1993): RAPD (random amplied polymorphic DNA) analysis based intervarietal genetic relationships among hexaploid wheats. - Plant Science 93: 95-103.

[29] Kalappanavar, I. K., Patidar, R. K., Srikant, K. (2008): Management strategies of leaf rust of wheat caused by Puccinia recondita f. Sp. Triticirob. Ex. Desm. - Karnataka Journal of Agricultural Sciences 21(1): 61-64.

[30] Kamyab, M., Hassani, H., Tohidinejad, E. (2009): Agronomic behavior of a new cereal (Tritipyrum: AABBEbEb) compared with modern Triticale and Iranian bread wheat cultivars. - Plant Echophysiology 150: 69-80.

[31] Kandel, J. S., Krishnan, V., Jiwan, D., Chen, X., Skinner, D. Z., See, D. R. (2017): Mapping genes for resistance to stripe rust in spring wheat landrace PI 480035. - PloS One 12(5): p.e0177898.

[32] Khan, M. A., Hussain, M., Hussain, M. (2002): Wheat leaf rust (Puccinia recondita) occurrence and shifts in its virulence in Punjab and NWFP. - Pakistan Journal of Phytopathology 14: 1-6.

[33] Kim, H. S., Ward, R. W. (2000): Patterns of RFLP-based genetic diversity in germplasm pools of common wheat with different geographical or breeding program origins. Euphytica 115: 197-208.

[34] Kraic, F., Mocák, J., Roháčik, T., Sokolovičová, J. (2009): Chemometric characterization and classification of new wheat genotypes. - Nova Biotechnol 9: 101-106.

[35] Lagudah, E. S., McFadden, H., Singh, R. P., Huerta-Espino, J., Bariana, H. S., Spielmeyer, W. (2006): Molecular genetic characterisation of the Lr34/Yr18 slow rusting resistance gene region in wheat. - Theoretical and Applied Genetics 114: 21-30.

[36] Lagudah, E. S., Krattinger, S. G., Herrera-Foessel, S., Singh, R. P., Huerta-Espino, J., Spielmeyer, W., Brown-Guedira, G., Selter, L. L., Keller, B. (2009): Gene-specific markers for the wheat gene $\operatorname{Lr} 34 / \mathrm{Yr} 18 / \mathrm{Pm} 38$ which confers resistance to multiple fungal pathogens. - Theoretical and Applied Genetics 119: 889-898.

[37] Leilah, A. A., Al-Khateeb, S. A. (2005): Statistical analysis of wheat yield under drought conditions. - Elsevier 61: 483-496.

[38] Mellingers, J. S. (1972): Measures of genetic similarity and genetic distance. - Studies in Genetics VII Univ Tex Publ. 27(13): 145-153.

[39] Muhammadi, S. A., Prasanna, B. M. (2003): Analysis of genetic diversity in crop plantsSalient statistical tools and considerations. - Crop Science 43: 1234-1248.

[40] Mustafa, G., Alam, M. M., Khan, S. U., Naveed, M., Mumtaz, A. S. (2013): Leaf rust resistance in semi dwarf wheat cultivars: a conspectus of post green revolution period in Pakistan. - Pakistan Journal of Botany 45: 415-422.

[41] Mutari, B., Udupa, S. M., Mavindidze, P., Mutengwa, C. S. (2018): Detection of rust resistance in selected Zimbabwean and ICARDA bread wheat (Triticum aestivum) germplasm using conventional and molecular techniques. - South African Journal of Plant and Soil 35(2): 101-10.

[42] Nagaoka, T., Ogihara, Y. (1997): Applicability of inter-simple sequence repeat polymorphisms in wheat for use as DNA markers in comparison to RFLP and RAPD markers. - Theoretical and Applied Genetics 94: 597-602. 
[43] Patpour, M., Hovmoller, M., Justesen, A., Newcomb, M., Olivera, P., Jin, Y., Szabo, L., Hodson, D., Shahin, A., Wanyera, R. (2016): Emergence of virulence to SrTmp in the Ug99 race group of wheat stem rust, Puccinia graminis f. sp. tritici, in Africa. - Frontier in Plant Science 7: 34-45.

[44] Peterson, R. F., Campbell, A. B., Hannah, A. E. (1948): A diagrammatic scale for estimating rust severity on leaves and stems of cereals. - Canadian Journal of Genetics and Cytology 26: 496-500.

[45] Prasad, M., Varshney, R. K., Roy, J. K., Balyan, H. S., Gupta, P. K. (2000): The use of microsatellites for detecting DNA polymorphism, genotype identification and genetic diversity in wheat. - Theoretical and Applied Genetics 100: 584-592.

[46] Pretorius, Z., Visser, B., Terefe, T., Herselman, L., Prins, R., Soko, T., Siwale, J., Mutari, B., Selinga, T., Hodson, D. (2015): Races of Puccinia triticina detected on wheat in Zimbabwe, Zambia and Malawi and regional germplasm responses. - Australian Journal of Plant Pathology 44: 217-224.

[47] Rao, S. K. V., Snow, J. P., Berggren, G. T. (1989): Effect of growth stages and initial inoculum level on leaf rust development and yield losses caused by Puccinia recondita $\mathrm{f}$. sp. tritici. - Journal of Phytopathology 127: 200-210.

[48] Rehman, A. U., Sajjad, M., Khan, S. H., Ahmad, N. (2013): Prospects of wheat breeding for durable resistance against brown, yellow and black rust fungi. - International Journal of Agriculture and Biology 15: 1209-1220.

[49] Roder, M. S., Korzun, V., Gill, B. S., Ganal, M. W. (1998): The physical mapping of microsatellite markers in wheat. - Genome 41: 278-283.

[50] Roelfs, A. P. (1988): Genetic control of phenotypes in wheat stemrust. - Annual Review of Phytopathology 26: 351-367.

[51] Roelfs, A. P., Singh, R. P., Saari, E. E. (1992): Rust Diseases of Wheat: Concepts and Methods of Disease Management. - CIMMYT, D. F., Mexico.

[52] Singh, R. P., Mujeeb-Kazi, A., Huerta-Espino, J. (1998): Lr46: a gene conferring slow rusting resistance to leaf rust in wheat. - Phytopathology 88(9): 890-894.

[53] Singh, R. P., Huerta-Espino, J., Rajaram, S. (2000): Achieving near-immunity to leaf rust and stripe rust in wheat by combining slow rusting resistance genes. - Acta Phytopathologica et Entomologica Hungarica 35: 133-139.

[54] Singh, R. P., Huerta-Espino, J., William, H. M. (2005): Genetics and breeding for durable resistance to leaf and stripe rusts in wheat. - Turkish Journal of Agriculture and Forestry 29: 121-127.

[55] Singh, S., Bowden, R. L. (2011): Molecular mapping of adult-plant race-specific leaf rust resistance gene Lr12 in bread wheat. - Molecular Breeding 28: 137-142.

[56] Stachel, M., Lelly, T., Grausgruber, H., Vollmann. (2000): Application of microsatellites in wheat (Triticum aestivum L.) for studying genetic differentiation caused by selection for adaptation and use. - Theoretical and Applied Genetics 100: 242-248.

[57] Suenaga, K., Singh, R. P., Huerta-Espino, J. and William, H. M. (2003): Microsatellite markers for genes $\operatorname{Lr} 34 / \mathrm{Yr} 18$ and other quantitative trait loci for leaf rust and stripe rust resistance in bread wheat. - Phytopathology 93: 881-890.

[58] Terefe, T. G., Visser, B., Herselman, L., Selinga, T., Pretorius, Z. A. (2014): First report of Puccinia triticina (leaf rust) race FBPT on wheat in South Africa. - Plant Disease 98: 101-109.

[59] William, H. M., Singh, R. P., Huerta-Espino, J., Ortiz-Islas, S., Hoisington, D. (2003): Molecular Marker mapping of leaf rust resistance gene Lr46 and its association with stripe rust gene $\operatorname{Yr} 29$ in wheat. - Phytopathology 93: 153-159.

[60] Zhang, P., Dreisigacker, S., Melchinger, A. E., Reif, J. C., Ginkel, M. V., Kazi, A., Hoisington, D., Warburton, M. L. (2005): Quantifying novel sequence variation and selective advantage in synthetic hexaploid wheats and their backcross-derived lines Using SSR markers. - Molecular Breeding 15: 1-10. 